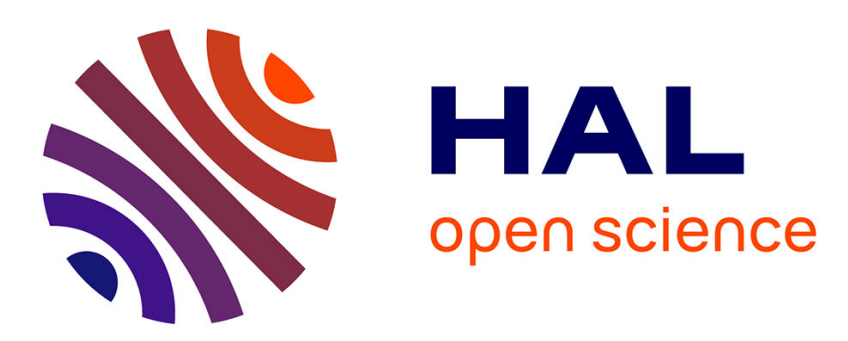

\title{
Analyse stœechiométrique de couches évaporées d'oxyde de silicium par observation simultanée des réactions nucléaires $(d, p)$ sur l'oxygène et le silicium
}

\author{
A. Cachard, J. Pivot, A. Roger, M. Talvat, J.-P. Thomas
}

\section{- To cite this version:}

A. Cachard, J. Pivot, A. Roger, M. Talvat, J.-P. Thomas. Analyse stœchiométrique de couches évaporées d'oxyde de silicium par observation simultanée des réactions nucléaires $(\mathrm{d}, \mathrm{p})$ sur l'oxygène et le silicium. Revue de Physique Appliquée, 1971, 6 (3), pp.279-283. 10.1051/rphysap:0197100603027900 . jpa-00243542

\section{HAL Id: jpa-00243542 https://hal.science/jpa-00243542}

Submitted on 1 Jan 1971

HAL is a multi-disciplinary open access archive for the deposit and dissemination of scientific research documents, whether they are published or not. The documents may come from teaching and research institutions in France or abroad, or from public or private research centers.
L'archive ouverte pluridisciplinaire HAL, est destinée au dépôt et à la diffusion de documents scientifiques de niveau recherche, publiés ou non, émanant des établissements d'enseignement et de recherche français ou étrangers, des laboratoires publics ou privés. 


\title{
ANALYSE STCCHIOMÉTRIQUE DE COUCHES ÉVAPORÉES D'OXYDE DE SILICIUM PAR OBSERVATION SIMULTANÉE DES RÉACTIONS NUCLÉAIRES (d, p) SUR L'OXYGẼNE ET LE SILICIUM
}

\author{
A. CAChARD $(*)$, J. PIVOT (*), A. ROGER $(*)$, \\ M. TALVAT $(* *)$ et J.-P. THOMAS $(* *)$ \\ Université Claude-Bernard, Lyon I, 43, bd du 11-Novembre-1918, \\ 69 , Villeurbanne
}

(Reçu le 23 décembre 1970)

\begin{abstract}
Résumé. - La microanalyse par observation directe de réactions nucléaires a été appliquée au dosage de l'oxygène 16 et du silicium 28 . Les spectres caractéristiques des réactions $(\mathrm{d}, \mathrm{p})$ sur ces

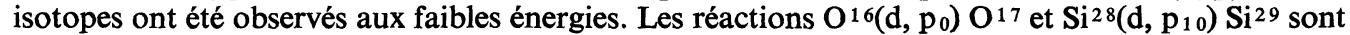
suffisamment séparées pour permettre le dosage simultané de ces deux éléments. L'énergie des deutons incidents la plus favorable est de $1,5 \mathrm{MeV}$ et la précision sur le rapport $N_{\mathrm{o}} / N_{\mathrm{si}}$ est meilleure que $1 \%$. Nous avons utilisé cette méthode pour l'étude de la stœchiométrie d'oxydes de silicium évaporés, et nous avons pu suivre l'influence de la pression résiduelle et de la vitesse d'évaporation sur la composition du dépôt. Cette technique peut également être employée pour l'analyse de la stœchiométrie d'autres composés en couches minces tels que l'alumine ou le nitrure de silicium.

Abstract. - Microanalysis by the direct observation of nuclear reactions is applied to the dosing of oxygen 16 and silicon 28 . The characteristic spectra of $(d, p)$ reactions on these isotopes are observed at low energies. $\mathrm{O}^{16}\left(\mathrm{~d}, \mathrm{p}_{0}\right) \mathrm{O}^{17}$ and $\mathrm{Si}^{28}\left(\mathrm{~d}, \mathrm{p}_{10}\right) \mathrm{Si}^{29}$ reactions are separated enough to allow the simultaneous dosing of these compounds. The most convenient energy of incident deuterons is $1.5 \mathrm{MeV}$ and the accuracy of $N_{\mathrm{o}} / N_{\mathrm{si}}$ ratio is better than $1 \%$. This method is used to study the stoechiometry of evaporated silicon oxides. The residual pressure and evaporation rate influence on the composition of the deposit is observed. This method can be used to analyse the stoechiometry of other thin film materials such as alumina and silicon nitride.
\end{abstract}

Introduction. - Les phénomènes de conduction dans les couches d'isolant amorphes font l'objet de nombreux travaux. Le plus souvent, il s'agit d'oxydes qui se présentent sous la forme d'un composé assez mal défini. L'écart à la stœchiométrie modifie alors profondément les propriétés diélectriques et le comportement électronique. $\mathrm{Ce}$ fait est particulièrement sensible pour l'oxyde de silicium évaporé "SiO ». Il était donc intéressant de posséder une technique permettant d'analyser de façon précise un tel composé sous forme de couche mince, c'est-à-dire pour une très faible quantité de matière.

Les méthodes utilisables sont essentiellement les microanalyseurs à sonde électronique ou ionique, les méthodes chimiques et les méthodes nucléaires.

Le microanalyseur à sonde électronique [1], [2] permet la détection d'éléments légers comme l'oxygène et le silicium. Cependant, pour un dosage quantitatif, cette technique semble peu appropriée car la masse de matière excitée n'est pas connue et les

(*) Département de Physique des Matériaux, Laboratoire d'Emission Electronique.

(**) Institut de Physique Nucléaire, Laboratoire de Chimie Nucléaire. corrections à apporter aux intensités mesurées ne sont pas encore bien définies [3].

La microanalyse par émission ionique secondaire [4], [5] permet une analyse chimique locale; tous les éléments sont décelables quel que soit leur numéro atomique, et la sensibilité est élevée. Mais le rendement d'ionisation dépendant fortement de la structure cristalline, l'interprétation quantitative des résultats reste encore délicate.

Pour le cas particulier de l'oxyde $\mathrm{SiO}_{x}$, Ritter [6] a indiqué une méthode d'analyse chimique. On réduit des ions argent à l'état argent élémentaire à l'aide des ions silicium incomplètement oxydés présents dans la couche. La masse totale est calculée à partir de la mesure de l'épaisseur par interférométrie et la composition en est déduite en comparant avec la masse réduite équivalente. Cependant la multiplication des mesures rend cette méthode relativement peu précise.

Nous avons préféré utiliser la microanalyse par observation directe de réactions nucléaires, méthode essentiellement développée par Amsel et ses collaborateurs [7], [8], [9]. Cette analyse consiste à observer les particules chargées (protons, rayons $\alpha$ ) émises lors du bombardement d'un échantillon par un fais- 
ce au de deutons de faible énergie $(<2 \mathrm{MeV})$. L'énergie des particules détectées est caractéristique du noyau bombardé. Le spectre en énergie, obtenu au moyen d'un détecteur à semi-conducteur, permet de déterminer la nature des éléments présents dans l'échantillon sur une profondeur de plusieurs microns, et par comparaison avec un étalon, de les doser quantitativement. C'est ainsi que l'oxygène et l'azote ont été dosés par Amsel [10] dans des couches d'oxyde anodique de tantale. L'intérêt du présent travail consiste à montrer qu'il est possible de faire un dosage simultané des éléments $\mathrm{O}$ et $\mathrm{Si}$, et notamment de calculer avec une très bonne précision le rapport $y / x$ du composé $\mathrm{Si}_{x} \mathrm{O}_{y}$.

La figure 1 donne le schéma de principe et la géométrie de l'appareillage.

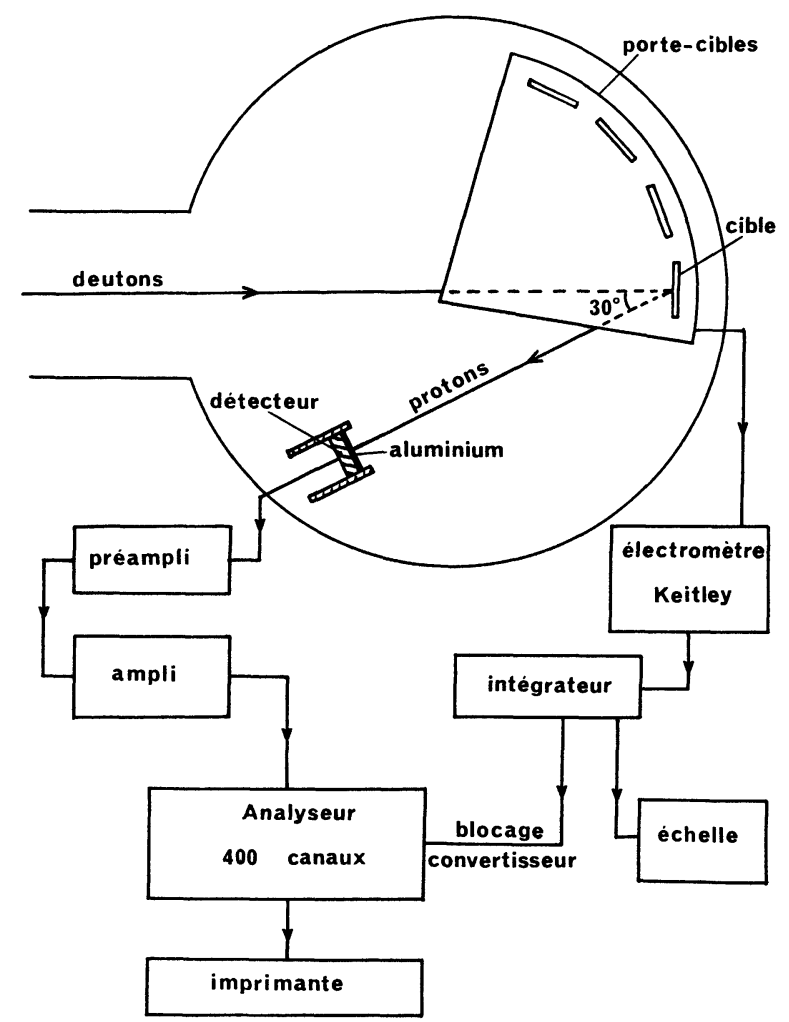

Fig. 1. - Schéma de principe et géométrie de l'appareillage.

I. Préparation des échantillons. - Nous désirons analyser des couches minces d'oxyde de silicium évaporées à partir du monoxyde commercial, soit par chauffage par effet Joule dans un creuset, soit par chauffage par bombardement électronique. De plus, nous désirons suivre l'évolution de leur composition stœchiométrique en fonction des conditions de préparation : pression résiduelle et vitesse d'évaporation.

Les échantillons sont évaporés soit dans une installation à vide ionique, soit dans une installation à vide classique (pompe à diffusion d'huile). Leur épaisseur, toujours inférieure à $0,5 \mu$, est mesurée par interférométrie (méthode de Nomarski). Elle est suivie en cours de fabrication par une microbalance à quartz qui permet également de contrôler la vitesse d'évaporation.
CHOIX DU SUPPORT. - Le support doit répondre à plusieurs impératifs. Il ne doit pas contenir d'oxygène ni de silicium; il ne doit pas être oxydable afin de pouvoir recuire l'échantillon; il doit être conducteur pour évacuer les charges électriques lors du bombardement par les deutons; enfin, il ne doit pas contenir d'éléments susceptibles de fournir des réactions nucléaires parasites.

Après quelques essais, nous avons choisi des disques d'or de $3 / 10 \mathrm{~mm}$ d'épaisseur. Nous avons en effet vérifié que l'adsorption d'oxygène lors des divers traitements reste négligeable par rapport à la quantité de cet élément présente dans la couche d'oxyde. Cependant, l'utilisation de l'or, élément lourd, entraîne une rétrodiffusion importante des deutons incidents. Ces particules sont éliminées en plaçant devant le détecteur un film mince d'aluminium (épaisseur massique $5,2 \mathrm{mg} / \mathrm{cm}^{2}$ ).

II. Réactions utilisées. - Les réactions nucléaires utilisables pour un dosage simultané doivent satisfaire à certaines conditions, notamment avoir une section efficace élevée et avoir des valeurs de $Q$ permettant d'obtenir des pics de détection bien séparés.

Pour le dosage de l'oxygène, on utilise dans la plupart des cas la réaction $\mathrm{O}^{16}(\mathrm{~d}, \mathrm{p}) \mathrm{O}^{17 *}$, car elle présente une section efficace permettant de faire des mesures précises [8].

Le silicium n'a été que peu étudié aux basses énergies. Cependant, Amsel [9] a utilisé les réactions $\mathrm{Si}^{29}(\mathrm{~d}, \alpha) \mathrm{Al}^{27} *$ et $\mathrm{Si}^{30}(\mathrm{~d}, \alpha) \mathrm{Al}^{28}$ pour suivre l'incorporation de silicium à la surface d'échantillon de zirconium soumis à des traitements mécaniques divers (polissage). Dans notre cas, l'utilisation de telles réactions est exclue car le film d'aluminium placé devant le détecteur absorbe le rayonnement.

Nous avons donc essayé d'étendre au silicium l'utilisation des réactions $(\mathrm{d}, \mathrm{p})$. Ces réactions ont fait l'objet d'une étude aux faibles énergies (entre 1 et $3 \mathrm{MeV}$ ) par Descroix [11] de laquelle il résulte que les réactions exploitables sont essentiellement : $\mathrm{Si}^{28}\left(\mathrm{~d}, \mathrm{p}_{3}\right) \mathrm{Si}^{29}, \mathrm{Si}^{28}\left(\mathrm{~d}, \mathrm{p}_{4}\right) \mathrm{Si}^{29}, \mathrm{Si}^{28}\left(\mathrm{~d}, \mathrm{p}_{10}\right) \mathrm{Si}^{29}$.

Nous avons choisi d'utiliser la réaction $\mathrm{Si}^{28}\left(\mathrm{~d}, \mathrm{p}_{10}\right) \mathrm{Si}^{29}$ car elle correspond à la section efficace la plus importante : cette dernière est de l'ordre de grandeur de celles des réactions $(d, p)$ sur l'oxygène, et dix fois plus grande que pour les réactions $\left(d, p_{3}\right)$ et $\left(\mathrm{d}, \mathrm{p}_{4}\right)$. Le tableau $\mathrm{I}$ résume les réactions intéressantes pour l'oxygène et le silicium avec les valeurs correspondantes de $Q$.

\section{TABLEAU I}

$\begin{array}{ccc} & \text { Etat du } & \\ \text { Réactions } & \text { noyau résiduel } & Q(\mathrm{MeV}) \\ \mathrm{O}^{16}(\mathrm{~d}, \mathrm{p}) \mathrm{O}^{17} & - & - \\ & \text { fondamental } & 1,919 \\ & \text { 1er état excité } & 1,048 \\ \mathrm{Si}^{28}(\mathrm{~d}, \mathrm{p}) \mathrm{Si}^{29} & \text { fondamental } & 6,253 \\ & 3^{\mathrm{e} \text { état excité }} & 3,83 \\ & \text { 4e état excité } & 3,187 \\ & \text { 10e état excité } & 1,32\end{array}$


Pour mesurer la valeur globale du rapport $N_{\mathrm{O}} / N_{\mathrm{Si}}$ par une analyse simultanée des réactions sur l'oxygène et le silicium, la condition nécessaire est l'obtention d'un spectre d'énergie présentant des pics nettement séparés pour $\mathrm{O}^{16}$ et $\mathrm{Si}^{28}$, et non perturbés par des réactions parasites telles que $\mathrm{C}^{12}\left(\mathrm{~d}, \mathrm{p}_{0}\right) \mathrm{C}^{13}$ ou $\mathrm{D}(\mathrm{d}, \mathrm{p}) \mathrm{T}$.

La figure 2 représente la position en énergie du proton provenant des différentes réactions en fonction de l'énergie des deutons incidents. Il apparaît

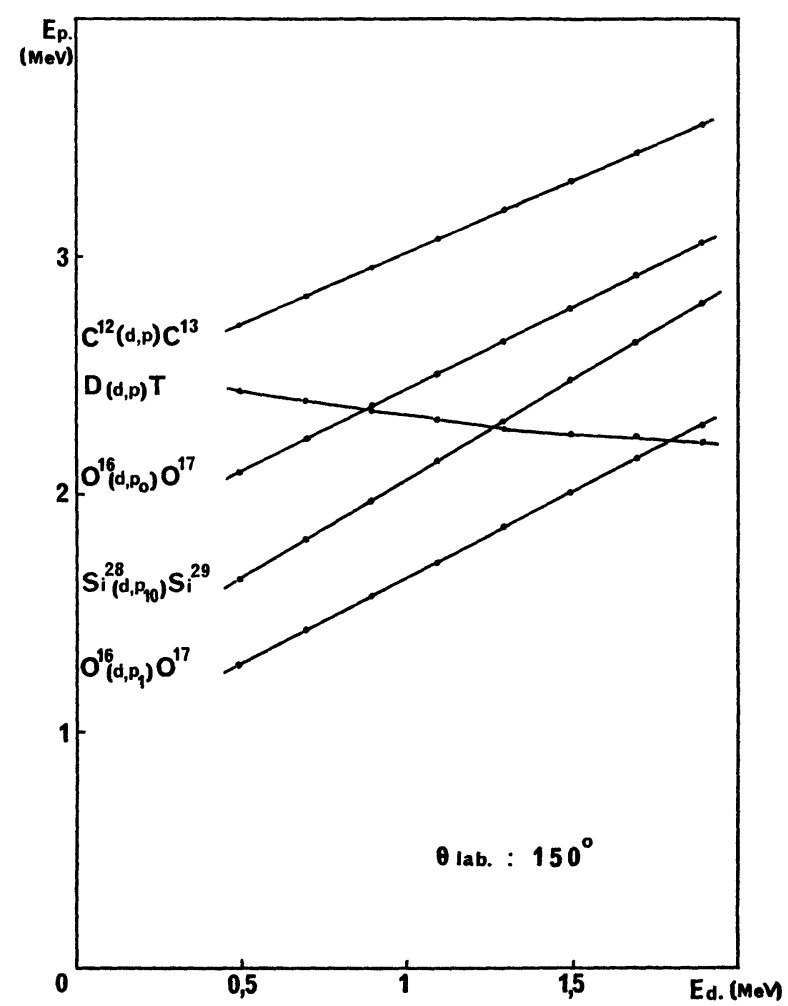

Fig. 2. - Energie de la particule émise en fonction de l'énergie des deutons incidents. $\theta_{\mathrm{lab}}=150^{\circ}$.

alors que la réaction la plus gênante sera $\mathrm{D}(\mathrm{d}, \mathrm{p}) \mathrm{T}$. On en déduit que les énergies optimales sont :

$$
\begin{aligned}
& E \mathrm{~d} \leqslant 0,700 \mathrm{MeV} \\
& E \mathrm{~d} \simeq 1,1 \mathrm{MeV}
\end{aligned}
$$

et

$$
1,5 \leqslant E \mathrm{~d} \leqslant 1,6 \mathrm{MeV}
$$

De plus, les résultats de Descroix et nos essais, montrent que la section efficace de la réaction $\mathrm{Si}^{28}\left(\mathrm{~d}, \mathrm{p}_{10}\right) \mathrm{Si}^{29}$ augmente de façon à peu près monotone jusqu'à $2 \mathrm{MeV}$. Pour l'oxygène, la réaction $(d, p)$ présente un palier de section efficace entre 1,3 et $1,5 \mathrm{MeV}$ avec variations rapides au-delà [12]. Ces considérations nous ont amenés à choisir la valeur $E \mathrm{~d}=1,5 \mathrm{MeV}$.

La figure 3 donne un spectre caractéristique obtenu dans ces conditions avec une couche de SiO d'épaisseur $4000 \AA$ évaporée au canon à électrons. On remarquera la très faible pollution par le carbone : ceci est rendu possible par l'emploi du pompage ionique pour la chambre de mesures comme pour le dispositif d'évaporation. La réaction parasite $\mathrm{D}(\mathrm{d}, \mathrm{p}) \mathrm{T}$ n'apparaît pas ici grâce au temps de comptage réduit.

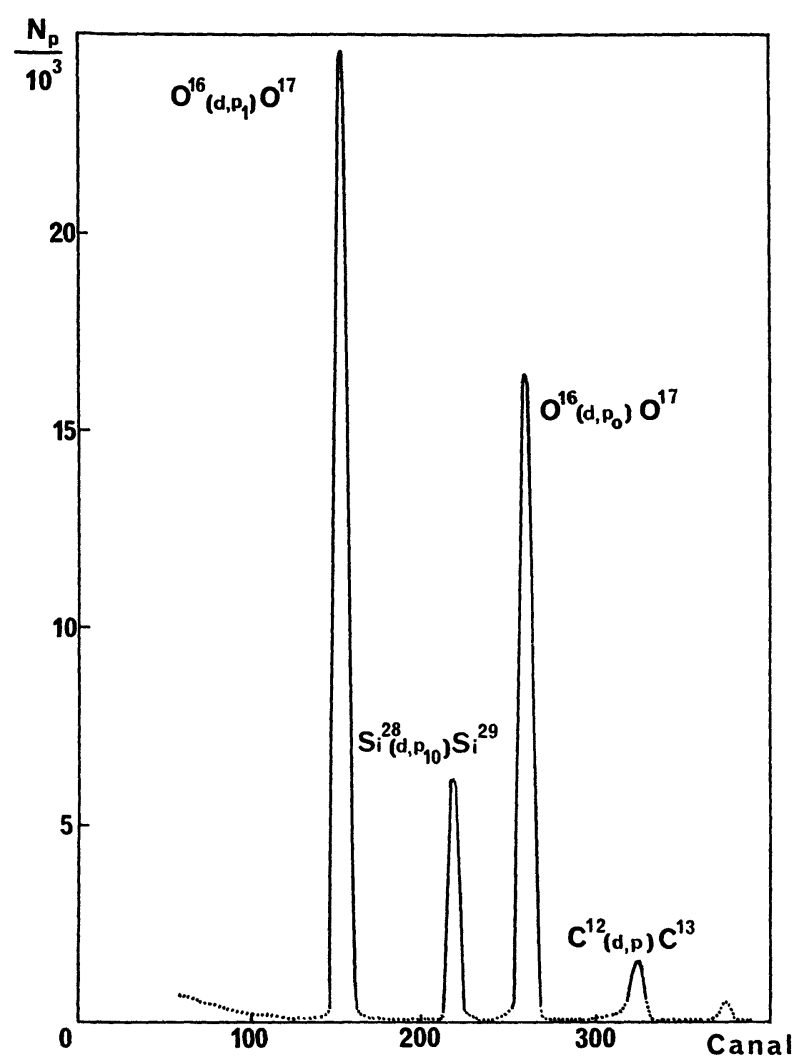

Fig. 3. - Spectre correspondant à une cible de monoxyde de silicium de 4000 Å évaporé avec un canon à électrons, bombardée par des deutons de 1,5 MeV. $9 \mathrm{keV}$ par canal.

A partir d'un tel spectre, le rapport $N_{\mathrm{O}} / N_{\mathrm{Si}}$ est calculé en faisant le rapport des pics intégrés pour les réactions $\left(d, p_{0}\right)$ sur $O^{16}$ et $\left(d, p_{10}\right)$ sur $S^{28}$. On choisit la réaction $\mathrm{O}^{16}\left(\mathrm{~d}, \mathrm{p}_{0}\right) \mathrm{O}^{17}$ de préférence à la réaction $\mathrm{O}^{16}\left(\mathrm{~d}, \mathrm{p}_{1}\right) \mathrm{O}^{17 *}$ car cette dernière risque d'être perturbée par des particules de faibles énergies constituant un fond parasite. La comparaison avec un étalon fournit alors la composition $N_{\mathrm{O}} / N_{\mathrm{Si}}$ réelle pour l'échantillon.

III. Cibles de référence et précision - L'oxyde stable de silicium est la silice (amorphe) ou le quartz (cristal) dont la composition correspond à la formule $\mathrm{SiO}_{2}$. En couche mince on peut fabriquer un tel oxyde, par exemple en oxydant du silicium à haute température sous oxygène. On doit alors, pour obtenir un étalon, séparer l'oxyde du silicium ou oxyder complètement une couche mince de silicium.

Nous avons utilisé une technique différente qui fournit un oxyde $\mathrm{SiO}_{2}$ de qualité équivalente comme l'ont indiqué Ramsey et Rice [13]. On évapore de la silice à l'aide d'un canon à électrons et on densifie cette couche par un recuit à $600^{\circ} \mathrm{C}$ à l'air pendant 18 heures. On obtient alors une couche $\mathrm{SiO}_{2}$ présen- 
tant le même spectre d'absorption infrarouge qu'un oxyde thermique, sans trace d'eau absorbée. L'analyse nucléaire révèle d'ailleurs que le recuit entraîne une diminution du rapport $N_{\mathrm{O}} / N_{\mathrm{Si}}$, ce qui correspond à l'élimination de la vapeur d'eau.

Un tel échantillon peut alors être pris comme étalon de la composition $\mathrm{SiO}_{2}$.

L'analyse consistant en des mesures comparatives, la précision n'est limitée que par la statistique. Généralement nous analysons des couches d'oxydes de $4000 \AA$ en faisant un comptage de l'ordre de 15 minutes avec un courant faisceau de $1 \mu \mathrm{A}$. Dans ces conditions, les pics intégrés dépassent largement 10000 coups, et la précision sur le rapport $N_{\mathrm{O}} / N_{\mathrm{Si}}$ est meilleure que $1 \%$.

D'autre part, nous avons vérifié que l'impact du faisceau ne modifie pas l'échantillon : pour cela, nous avons fait plusieurs comptages successifs sur le même point et n'avons pas observé de variations du rapport $N_{\mathrm{O}} / N_{\mathrm{Si}}$ supérieures aux fluctuations statistiques.

La figure 4 montre la partie utile de deux spectres correspondant aux compositions extrêmes $\mathrm{SiO}$ et $\mathrm{SiO}_{2}$.

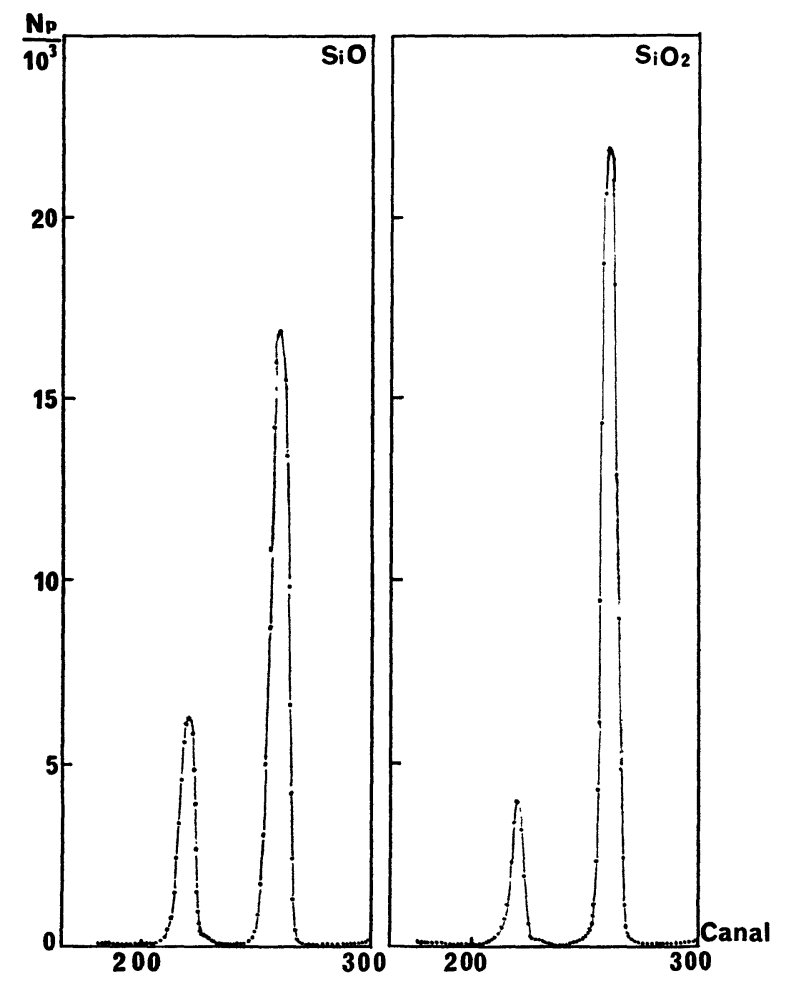

FIG. 4. - Partie utile du spectre correspondant aux deux compositions extrêmes $\mathrm{SiO}$ et $\mathrm{SiO}_{2}$.

IV. Résultats. - Nous avons utilisé la méthode d'analyse ainsi décrite pour déterminer la composition en oxygène et en silicium (rapport $N_{\mathrm{o}} / N_{\mathrm{Si}}$ ) d'oxydes de ce métal en fonction des conditions d'évaporation. Nous figurerons ces conditions par le paramètre $p / r$, où $p$ est la pression résiduelle exprimée en
$10^{-6}$ torr et $r$ la vitesse d'évaporation en $\AA / \mathrm{s}$. Ce paramètre représente approximativement le rapport des nombres de molécules $\mathrm{O}_{2}$ et $\mathrm{SiO}$ arrivant sur le support en cours d'évaporation [14].

La figure 5 donne les variations de $N_{\mathrm{O}} / N_{\mathrm{Si}}$ en fonction de $p / r$. Nous constatons que pour les évaporations au moyen d'un canon à électrons (SiO canon) la stœchiométrie correspond toujours au monoxyde SiO. Par contre, l'évaporation par effet Joule (SiO therm.) ne permet pas d'atteindre la stœchiométrie $N_{\mathrm{O}} / N_{\mathrm{Si}}=1$ dans le domaine de $p / r$ utilisé. L'oxyde se rapprochant le plus de cette valeur

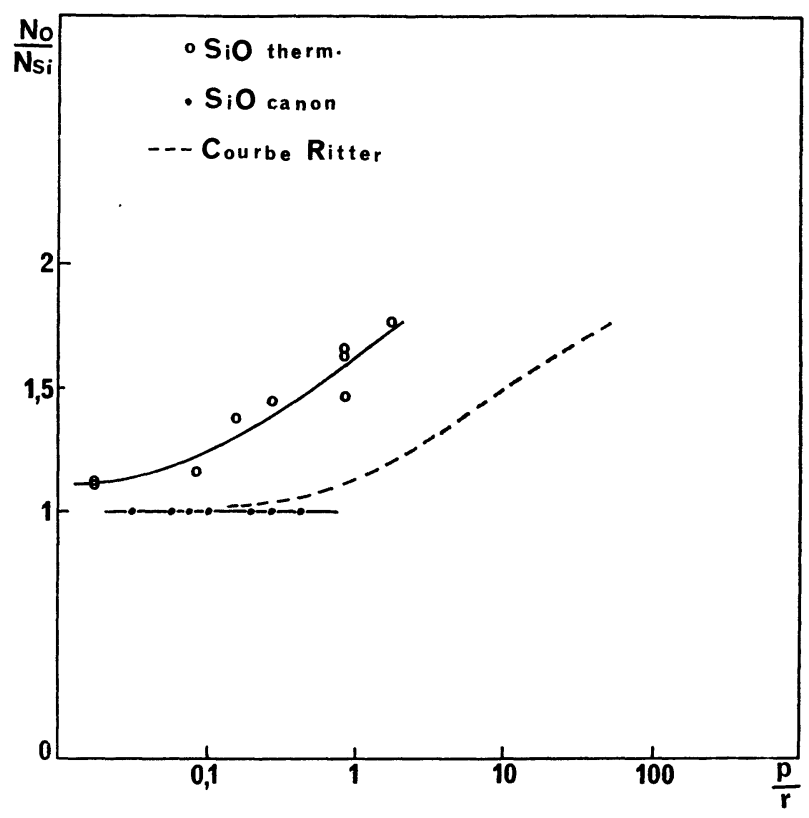

FIG. 5. - Rapport $N_{\mathrm{o}} / N_{\mathrm{Si}}$ en fonction de $p / r$. La courbe en pointillés correspond aux résultats de Ritter.

correspond à la composition $\mathrm{SiO}_{1,12}$ et le rapport $N_{\mathrm{o}} / N_{\mathrm{Si}}$ augmente rapidement. La courbe expérimentale que nous obtenons présente une grande analogie avec la courbe théorique de Ritter déduite de ses mesures [6]. Cependant, il existe un décalage d'un ordre de grandeur en $p / r$ qui peut avoir plusieurs origines :

- dans l'analyse chimique, le degré d'oxydation des ions silicium actifs n'est pas bien défini et la détermination de $N_{\mathrm{O}} / N_{\mathrm{Si}}$ semble donc moins précise qu'avec notre méthode,

- l'étalonnage des appareils de mesures de vide peut varier d'une façon non négligeable d'une installation à l'autre,

- les évaporations sont faites à des pressions plus faibles et pour des vitesses toujours $\leqslant 15 \AA /$ s. La température de la source et la teneur en vapeur d'eau de l'atmosphère résiduelle prennent alors une plus grande importance [15],

Les écarts entre les résultats obtenus avec un canon à électrons et ceux avec une source à effet Joule peuvent s'expliquer par une différence de la température des 
deux sources. En effet, par suite de la faible quantité de matière bombardée, une même vitesse d'évaporation correspond pour un $\mathrm{SiO}$ canon à une température de source plus élevée que pour un SiO thermique, ce qui entraîne une plus faible influence de l'atmosphère résiduelle [15]. Cet effet est renforcé ici par les faibles vitesses d'évaporations utilisées.

Conclusion. - Nous avons pu montrer que la méthode de microanalyse par observation simultanée des réactions nucléaires sur l'oxygène et le silicium permet de déterminer la composition globale d'un oxyde en couche mince $\mathrm{Si}_{x} \mathrm{O}_{y}$ avec une précision meilleure que $1 \%$ sur le rapport $y / x$. Pour un composé monocristallin où les "impuretés " (telles qu'un écart à la stœchiométrie), même à des concentrations de quelques p. p. m., jouent un rôle important, une telle précision serait insuffisante. Mais des études menées sur la conduction des matériaux amorphes montrent que celle-ci est peu sensible à la présence d'impuretés jusqu'à des concentrations de l'ordre de $1 \%$, ce qui justifie l'intérêt de notre étude.

Cette méthode présente en outre l'avantage de pouvoir s'appliquer aussi à la détermination de la composition d'autres matériaux d'un grand intérêt en métallurgie ou en micro-électronique, tels que l'alumine ou le nitrure de silicium.

Il faut remarquer que nous n'avons déterminé que la composition globale de l'échantillon. Une caractérisation plus complète nécessite l'emploi de mesures complémentaires comme l'absorption infrarouge, la résonance paramagnétique électronique ou les mesures des pertes diélectriques.

Remerciements. - Nous tenons à remercier Monsieur le Professeur J. Tousset et Monsieur le Professeur C. H. S. Dupuy d'avoir bien voulu suivre notre étude.

\section{Bibliographie}

[1] Guinier (A.) et Castaing (R.), C. R. Acad. Sci. Paris, 1951, 232, 1948.

[2] Castaing (R.), Advances Electronics Electron Physics, $1960,12,759$.

[3] Philibert (J.) et Tixier (R.), Brit. J. Appl. Phys. (J. Phys. D), 1968, Ser. 2, 1, 685

[4] Castaing (R.) et Slodzian (G.), C. R. Acad. Sci. Paris, 1962, 255, 1893.

[5] Slodzian (G.), Rev. Phys. Appl., 1968, 3, 360.

[6] RitTer (E.), J. Vac. Sci. Technol., 1966, 3, 225.

[7] Amsel (G.) et SAmuel (D.), Analytical Chemistry, $1967,39,1689$.

[8] Amsel (G.), David (D.), Beranger (G.) et Boisot (P.), Rev. Phys. Appl., 1968, 3, 373.
[9] Amsel (G.), David (D.), Beranger (G.), Boisot (P.), De Gelas (B.) et Lacombe (P.), J. Nuclear Materials, 1969, 29, 144.

[10] Amsel (G.), Cherki (C.), Feuillade (G.) et Nadai (J. P.), J. Phys. Chem. Solids, 1969, 30, 2117.

[11] Descroix (E.), Thèse Spécialité, Lyon, 1970.

[12] Amsel (G.), Ann. Phys., 1964, 9, 297.

[13] Ramsey (T. H.), et Rice (S. B.), J. Am. Ceram. Soc., 1969, 52, 225.

[14] Anastasio (T. A.), J. Appl. Phys., 1967, 38, 2606.

[15] Berry (R. W.), Hall (P. M.) et Harris (M. T.), Thin Film Techn., 1968, D. Van Nostrand Company Inc., Princeton, 155. 